\title{
Análisis de las relaciones económicas y comerciales entre Ecuador e Irán en el contexto de la geopolítica mundial
}

\author{
Analysis of economic and trade relations between \\ Ecuador and Iran in the global geopolitical context
}

PAMELA MONCAYO POZO

Consultora independiente

Correo electrónico: pame-moncayo@hotmail.com

JOSÉ SALGADO DEFRANC

Universidad Tecnológica Equinoccial

Correo electrónico: pepesalgadodefranc@yahoo.es

RECIBIDO: 01 septiembre 2014 / APROBADO: 30 octubre 2014

\section{Resumen}

Irán es un país con una historia milenaria, baste recordar las guerras médicas contra los griegos 500 años a. de n.e. En los albores de siglo XX, el país persa pasó a ser codiciado por potencias extranjeras debido a su ubicación geográfica, y al hecho de que posee grandes reservas de hidrocarburos. Desde el gobierno del economista Rafael Correa, Ecuador buscó un acercamiento con la nación iraní. El acercamiento no pudo concretarse en el plano comercial, en vista de que nuestro país hubiera recibido sanciones por parte de la comunidad internacional, que denuncia (sin que existan pruebas al respecto) de que Irán busca fabricar armas atómicas. Únicamente en el año 2010 Ecuador logró exportar cantidades algo significativas a Irán, exportaciones que han desaparecido en la actualidad. Respecto a inversiones iraníes en el Ecuador, en el período 2000-2010, estas apenas superaron los 20.000 dólares. Estos pocos datos indican que literalmente no existen relaciones económicas entre Ecuador e Irán. 
PALABRAS CLAVE: Ecuador, Irán, geopolítica, comercio exterior

\begin{abstract}
:
Iran is a country with an ancient history. Just remember the Persian Wars against the Greeks 500 years before our generation. At the beginning of the twentieth century this Persian country began to be coveted by foreign powers due to its geographical location, and due to the fact that it has large reserves of hydrocarbons. Since the government of Rafael Correa, Ecuador looked for an approach with the Iranian nation. The approach could not be realized in terms of trade, since our country would have been punished by the international community, which complains (without any supporting evidence) that Iran has the idea of building atomic weapons. Only in 2010 Ecuador got to export significant amounts of products to Iran, exports that have now disappeared. In relation to Iranian investments in Ecuador, in the period between 2000 and 2010 these investments reached just above 20,000 US dollars. These few data show that economic relations between Ecuador and Iran literally do not exist.
\end{abstract}

KEYWORDS: Ecuador, Iran, geopolitics, foreign trade

CLASIFICACIÓN JEL: : F40, F49.

\section{Metodología}

Para elaborar este ensayo se ha utilizado el método de la economía política que consiste en elevarse de lo concreto a lo abstracto y de lo abstracto a lo concreto. Se parte del concreto real, se lo volatiliza en un proceso de análisis, para en el proceso de la investigación alcanzar el concreto real, entendido como la síntesis de múltiples determinaciones.

\section{Irán en el contexto internacional del siglo $\mathrm{XX}$}

\subsection{Persia, el petróleo y la Primera Guerra Mundial}

Desde los inicios del siglo XX, Persia como era conocido Irán, fue objeto de disputas entre los países más poderosos del mundo por sus enormes reservas de crudo. Las primeras reservas de petróleo fueron descubiertas cerca de Ahwaz, en Juzistán, al sudoeste de Irán en 1908. Durante décadas, Persia, por su condición de país fronterizo con el noroeste de la India, había sido un enclave importante para Gran Bretaña debido a que las reservas petrolíferas de Juzistán se convirtieron en un elemento esencial para la seguridad del imperio británico. Gran Bretaña no tardó en ampliar su zona de influencia hacia el oeste y en adjudicarse el resto de la costa del golfo pérsico y los campos petrolíferos. Tras constituirse la Compañía Petrolífera Anglopersa (AIOC) en 1914, el gobierno británico se apropió de la mayoría de las acciones de la empresa.

Al final de la guerra, Irán era un país irreconocible. La contienda supuso la ruina del comercio y la producción agrícola iraníes, y 1917 y 1918 fueron años de hambruna. Para la balanza comercial, las 
consecuencias de la revolución rusa fueron también devastadoras: antes de 1914, el 65\% del comercio exterior iraní se destinaba a Rusia, pero, una vez que terminó la contienda mundial el porcentaje cayó al 5\% (AXWORTHY, 2010, pág. 251).

\subsection{La Revolución de 1979}

La Revolución islámica, que en 1979 supuso el final del régimen de Reza Pahlavi ${ }^{1}$, fue un acontecimiento inédito y sorprendente en la historia del siglo XX. En primer lugar, fue una revolución verdadera, en el sentido de un movimiento subversivo popular que fue capaz de derribar un régimen establecido, a diferencia de tantos golpes militares que, en naciones subdesarrolladas o en vías de desarrollo, tuvieron un resultado semejante pero sin la participación de las masas ni consecuencias tan radicales. Por otro lado, fue la primera ocasión en que el uso político del Islam desempeñó un papel absolutamente primordial y aun exclusivo, superando con mucho al que pudo tener en otro tiempo el nacionalismo de los países que habían superado el colonialismo.

A mediados de enero de 1979 pareció haberse iniciado un proceso hacia una monarquía constitucional. Al final, el sah abandonó Irán confiando el Gobierno a un dirigente en teoría occidentalista y socialdemócrata, Chapur Bakhtiar, cuyo poder se volatilizó en apenas diez días. El 11 de febrero de 1979, después de dos días enteros de motines y combates, la población sublevada junto con militares y guerrilleros favorables tomó por completo Teherán. Dos años antes, sin embargo, no existía ninguna fuerza de oposición organizada en Irán.

Muy pronto, sin embargo, se prohibieron las huelgas por cualquier tipo de causas, en otro tiempo promovidas por los izquierdistas. La Unión Soviética fue designada como "pequeño Satán" por Jomeini (el "gran Satán" serían los Estados Unidos) y, en vez de encargar la

1 La dinastía Pahlavi gobernó Irán gran parte del siglo XX. Primero fue Reza Shah Pahlavi, que reinó Irán entre 1925 y 1941, para ser sucedido por su hijo Mohammad Reza Shah Pahlavi, quien gobernó el país Persa entre 1941 y 1979. redacción de una nueva Constitución a una asamblea constituyente, se decidió que la llevara a cabo una reunión de expertos islámicos, la mayor parte de ellos muy próximos al Partido de la Revolución Islámica, que los seguidores del líder espiritual organizaron después de la expulsión del sah. En junio de 1989 murió Jomeini dejando una herencia importante a la Humanidad todavía no lo suficientemente estudiada y comprendida (SERRATO, 2009, pág. 221).

\section{Economía de Irán}

\subsection{Industria petrolera}

Las reservas de petróleo en Irán son las cuartas más grandes del mundo y así mismo es el cuarto productor mundial y el segundo productor de la OPEP después de Arabia Saudita. La información que presentamos a continuación está basada en cálculos realizados en el año $2010^{2}$.

\section{Tabla 1.}

Principales productores mundiales de petróleo

\begin{tabular}{ll}
\hline \multicolumn{1}{c}{ País } & Millones barriles \\
\hline 1. Arabia Saudita & 262,6 \\
\hline 2. Venezuela & 211,2 \\
\hline 3. Canadá & 175,2 \\
\hline 4. Irán & 137,0 \\
\hline 5. Iraq & 115,0 \\
\hline
\end{tabular}

Fuente: (CIA World Factbook, 2013)

A continuación se presenta en cambio los veinte principales productores de petróleo en la década pasada e importantes proyecciones para el año 2030. Para el caso que nos ocupa, podemos percatarnos que Irán en el 2030 seguirá ocupando el cuarto lugar en la producción mundial de este hidrocarburo.

\footnotetext{
2 Esta información tiene que tomarse como base para realizar cálculos actuales luego de que en EE.UU se ha desarrollado tecnologías para explotar el petróleo de esquisto
} 
Tabla 2.

Principales productores mundiales de petróleo a futuro

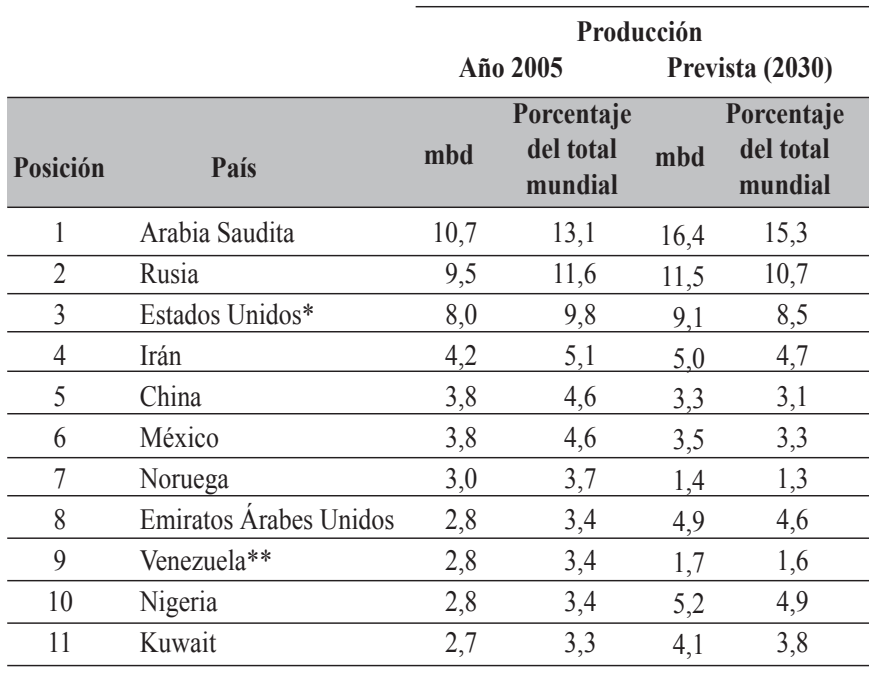

mbd: millones de barriles diarios

*Incluye liquido de gas natural

**No incluye petróleo extrapesado o líquidos derivados de arenas bituminosas

Fuente: (KLARE, 2008, pág. 69)

Tiene gran importancia geopolítica pasar a analizar a qué países del mundo Irán exporta su petróleo. La Tabla 3 recoge los países que más compraban a Irán:

Tabla 3.

Exportaciones de Irán 2012.

\begin{tabular}{ll}
\multicolumn{1}{c}{ País } & $\begin{array}{c}\text { Barriles de } \\
\text { petróleo }\end{array}$ \\
\hline China & 543.000 \\
\hline India & 341.000 \\
\hline Japón & 251.000 \\
\hline Italia & 249.000 \\
\hline Corea del sur & 239.000 \\
\hline Turquía & 217.000 \\
\hline España & 149.000 \\
\hline Grecia & 111.000 \\
\hline Sudáfrica & 98.000 \\
\hline Francia & 78.000 \\
\hline
\end{tabular}

Fuente: Central Bank of Irán (2012)

Luego de observar esta información, se puede percibir que con excepción de China y de la India el resto de países se ubican en la órbita de influencia de los EE.UU. Por tanto pasaron a formar parte del boicot que impuso los Estados Unidos a las exportaciones de petróleo de Irán desde el 2012, y que han sido levantadas desde inicios del 2014.

\subsection{Industria gasífera}

Si el petróleo tiene gran importancia para analizar el papel de Irán en la geopolítica mundial, más aún lo tiene las reservas de gas del país persa. A continuación se puede observar que Irán posee las segundas reservas más grandes de gas en el mundo. Se tiene que indicar también que solo tres países (Rusia, Irán y Qatar) poseen más del $50 \%$ de las reservas mundiales de gas. Revisemos información de Michael Klare al respecto.

\section{Tabla 4.}

Principales reservas mundiales de gas natural.

\begin{tabular}{|c|c|c|c|c|c|}
\hline \multirow[b]{2}{*}{ Pos. } & \multirow[b]{2}{*}{ País } & \multicolumn{3}{|c|}{ Reservas } & Producción \\
\hline & & Bpc & $\begin{array}{c}\text { Porcentaje } \\
\text { del total } \\
\text { mundial }\end{array}$ & mmpc & $\begin{array}{c}\text { Porcentaje } \\
\text { del total } \\
\text { mundial }\end{array}$ \\
\hline 1 & Rusia & $1.682,10$ & 26,3 & $21.607,10$ & 21,3 \\
\hline 2 & Irán & 993,00 & 15,5 & $3.706,50$ & 3,7 \\
\hline 3 & Qatar & 895,2 & 14,0 & $1.747,40$ & 1,7 \\
\hline 4 & Arabia Saudita & 249,7 & 3,9 & $2.601,60$ & 2,6 \\
\hline 5 & Emiratos Árabes Unidos & 214,0 & 3,3 & $1.673,20$ & 1,6 \\
\hline 6 & Estados Unidos & 209,2 & 3,3 & $18.500,70$ & 18,5 \\
\hline 7 & Nigeria & 189,9 & 2,9 & 995,50 & 1,0 \\
\hline 8 & Argelia & 159,0 & 2,5 & $2.982,90$ & 2,9 \\
\hline 9 & Venezuela & 152,3 & 2,4 & $1.013,10$ & 1,0 \\
\hline 10 & Iraq & 111,9 & 1,7 & \# & $\#$ \\
\hline 11 & Kazajistán & 105,9 & 1,7 & 843,70 & 0,8 \\
\hline 12 & Noruega & 102,1 & 1,6 & $3.092,30$ & 3,0 \\
\hline 13 & Turkmenistán & 101,0 & 1,6 & $2.195,70$ & 2,2 \\
\hline 14 & Indonesia & 92,9 & 1,5 & $2.612,20$ & 2,6 \\
\hline \multirow[t]{2}{*}{15} & Australia & 92,0 & 1,4 & $1.373,20$ & 1,4 \\
\hline & Total de las 15 & $5.350,20$ & 83,5 & $64.045,10$ & 64,2 \\
\hline
\end{tabular}

Nota: La suma total puede no ser exacta debido al redondeo. bpc: billones de pies cúbicos mmpc: miles de millones de pies cúbicos \#: insignificante

Fuente: (KLARE, 2008, pág. 70) 


\subsection{EI PIB PPA de Irán}

Como es conocido, el PIB calculado de acuerdo a la paridad del poder adquisitivo (PPA) elimina las distorsiones que generan los diferentes niveles de precios en todos los países del mundo. Al analizar el PIB PPA de todos los países del mundo nos encontramos con el hecho de que Irán ocupa el puesto número 17 a nivel mundial. Este dato desvirtúa la falsa creencia del atraso secular iraní. Utilizando este indicador se debe señalar que Irán en cuanto a su potencial económico se encuentra relativamente cerca de países como España y Canadá. (Central Bank of Iran, 2012).

\section{Tabla 5.}

PIB PPA de Irán al año 2012

\begin{tabular}{llc}
\hline $\mathbf{N}^{\mathbf{0}}$ & \multicolumn{1}{c}{ País } & $\begin{array}{c}\text { PIB PPA } \\
\text { (millones de USD) }\end{array}$ \\
\hline 1 & Estados Unidos & 15.609 .697 \\
\hline 2 & China & 12.387 .048 \\
\hline 3 & India & 4.824 .551 \\
\hline 4 & Japón & 4.588 .972 \\
\hline 5 & Alemania & 3.158 .090 \\
\hline 6 & Rusia & 2.510 .791 \\
\hline 7 & Brasil & 2.393 .954 \\
\hline 8 & Reino Unido & 2.308 .503 \\
\hline 9 & Francia & 2.257 .015 \\
\hline 10 & Italia & 1.834 .946 \\
\hline 11 & México & 1.743 .474 \\
\hline 12 & Corea Del Sur & 1.629 .904 \\
\hline 13 & Canadá & 1.443 .108 \\
\hline 14 & España & 1.405 .437 \\
\hline 15 & Indonesia & 1.208 .542 \\
\hline 16 & Turquía & 1.112 .265 \\
\hline 17 & Irán & 1.006 .540 \\
\hline
\end{tabular}

Fuente: Fondo Monetario Internacional, (2012)

\subsection{Industria automotriz}

La industria automotriz en Irán es la duodécima más grande en el mundo, su tasa de crecimiento de producción de automóviles es alta y se espera que llegue a ser una gran potencia. Irán produce algunos tipos de vehículos como furgonetas, camiones pesados, mini buses y motocicletas. Las compañías más importantes son Irán Khodro y Saipa. Ambas industrias tienen tecnologías de las empresas Peugeot y Citroen. Estas empresas también se dedican a la fabricación de partes y piezas como repuestos y a su vez son industrias donde se forman ingenieros automotrices (SOOFI \& GHAZINOORY, 2013, pág. 226).

\subsection{Inflación de Irán en los últimos años}

De acuerdo a la "tradición" de los países productores de petróleo del tercer mundo, en Irán una gran cantidad de productos se venden en el mercado con precios subsidiados. Debido a que una parte de estos subsidios se pagan con emisión inorgánica de dinero, el costo para la economía es el surgimiento de la inflación. En los últimos seis años la inflación acumulada ha sido de $315 \%$, siendo la inflación del 2008 al 2013 (ver Tabla 6.).

Tabla 6.

Inflación Irán 2008 al 2013

\begin{tabular}{cc}
\hline Años & Inflación \\
\hline 2008 & $25,60 \%$ \\
\hline 2009 & $13,50 \%$ \\
\hline 2010 & $31,50 \%$ \\
\hline 2011 & $20,60 \%$ \\
\hline 2012 & $27,10 \%$ \\
\hline 2013 & $42,30 \%$ \\
\hline
\end{tabular}

Fuente: (CIA World Factbook, 2013)

Otro factor que coadyuva en el proceso inflacionario iraní es el boicot a las exportaciones de petróleo impuesto por los EE.UU ${ }^{3}$.

\footnotetext{
3 Una clara comprensión de este fenómeno ameritaría otra investigación como la que estamos presentando.
} 


\section{Relaciones de Irán con el mundo}

La compleja relación de Irán con el mundo se debe a las grandes reservas de hidrocarburos que posee este país (como ya lo hemos visto en el transcurso de este artículo) y a su ubicación geográfica. En efecto Irán linda por un lado con el golfo Pérsico y por otro con el mar Caspio. Pasamos a continuación a analizar cada una de estas dos situaciones geográficas.

\subsection{Golfo Pérsico}

El golfo Pérsico, ubicado entre la península Arábiga y el mar Arábigo, ocupa la sexta parte de Asia y constituye la principal fuente energética del mundo. En la actualidad casi un $60 \%$ del petróleo que se comercializa en el mundo pasa por el golfo Pérsico y por el estrecho de Ormuz.

Figura 1.

Mapa del Golfo Pérsico

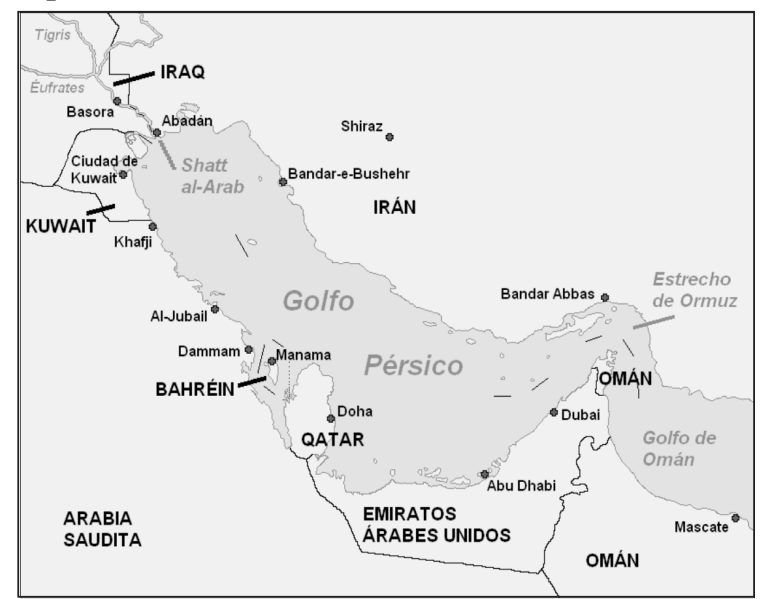

Fuente: (http://es.wikipedia.org/wiki/Golfo_Persico)

\subsection{El mar Caspio}

El mar Caspio no solo es importante por las grandes reservas de petróleo y de gas que posee, sino también por el hecho de tener fronteras con Rusia. Por esta razón para Rusia es una línea roja que no dejará ser tomada por Occidente. Gran parte del armamento estratégico que posee Irán ha sido entregado por Rusia.
Figura 2.

\section{Mapa del mar Caspio}

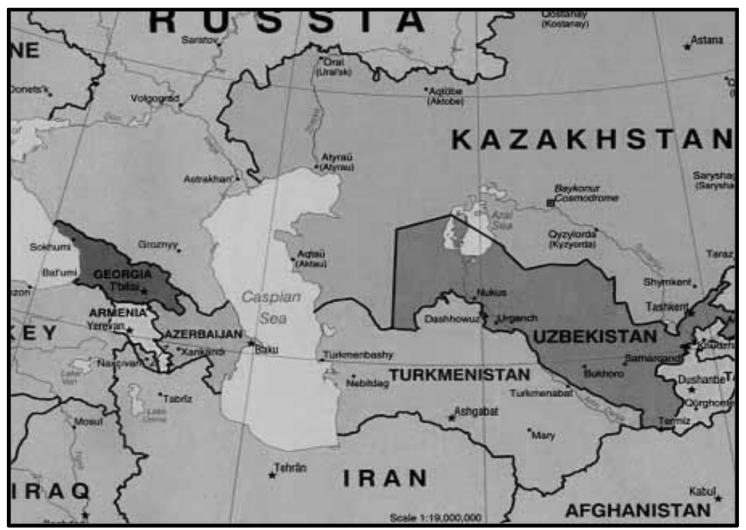

Fuente: (Enciclopedia Encarta, 2012)

\section{Relaciones comerciales entre Ecuador - Irán}

Las relaciones comerciales entre Ecuador e Irán se establecieron desde el actual Gobierno del economista Rafael Correa. En el año 2008 Ecuador e Irán restablecieron las relaciones diplomáticas. El Banco Central del Ecuador, ya en ese año, emitió un informe donde señala los riesgos y posibles sanciones para el Ecuador al participar en la implementación de un convenio para facilitar relaciones bilaterales entre Ecuador e Irán.

En el año 2009 se firmó un memorándum de entendimiento exploración mineray la implementación de un laboratorio químico - geotécnico - metalúrgico. En el año 2013 se firmó un acuerdo entre para el abastecimiento de combustibles a partir de 2013 (PROECUADOR, Ficha técnica Ecuador Iran, 2012).

\subsection{Balanza comercial Ecuador - Irán}

Para analizar las relaciones comerciales entre nuestro país y el persa, revisaremos los intercambios registrados en la balanza comercial petrolera y en la balanza comercial no petrolera. 
Figura 3.

Balanza comercial no petrolera Ecuador-Irán. Miles USD FOB

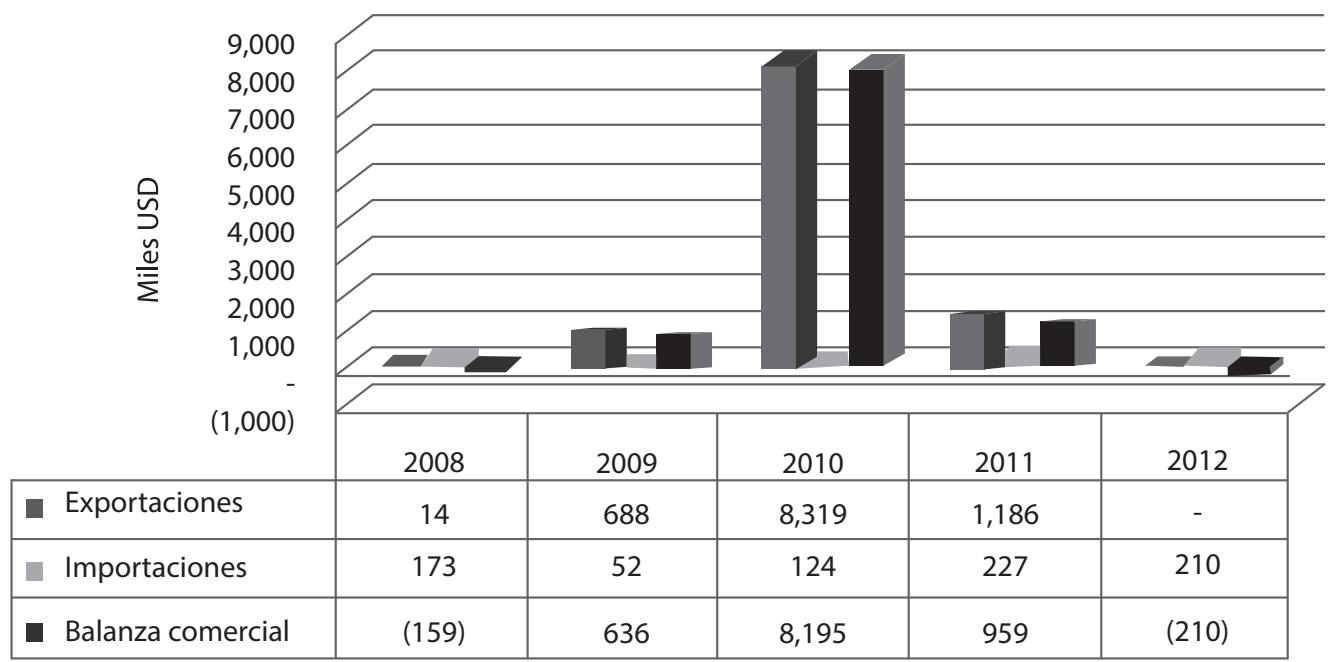

Fuente: (PROECUADOR, Ficha técnica Ecuador Iran, 2012)

\section{Figura 4.}

Balanza comercial petrolera Ecuador-Irán. Miles USD FOB

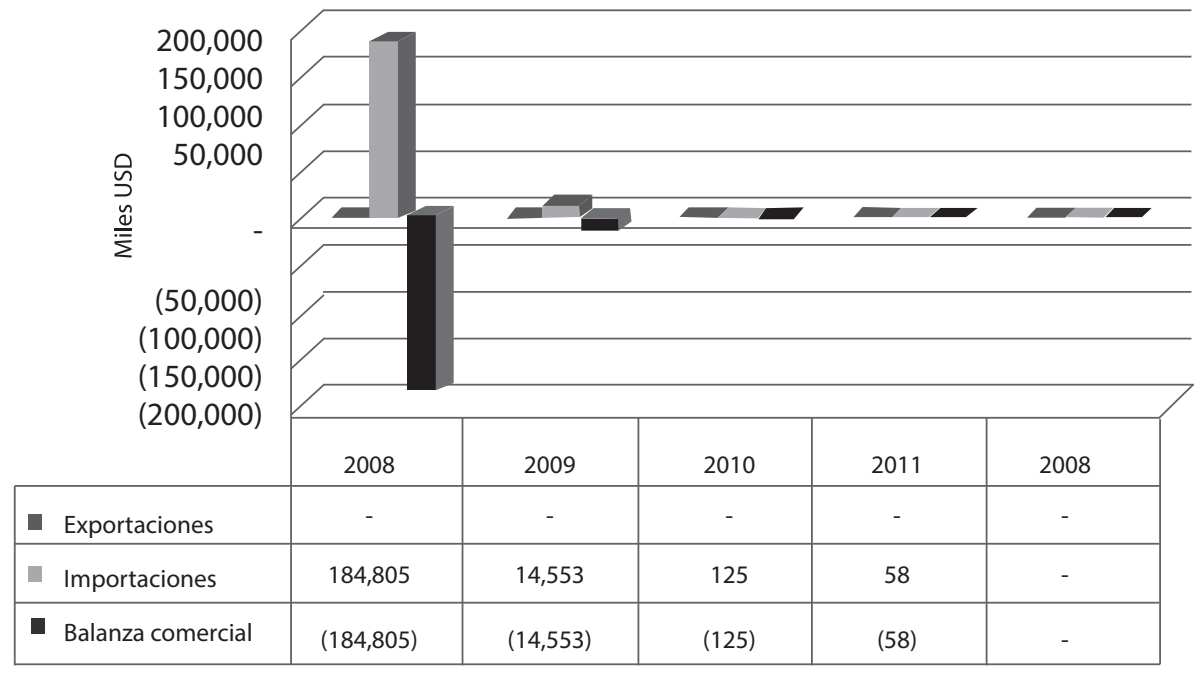

Fuente: (PROECUADOR, Ficha técnica Ecuador Iran, 2012)

Se puede observar que existe un comercio mínimo entre Ecuador e Irán. El banano representó (US\$ 8 millones aproximadamente) en el 2010 casi el $97 \%$ de la oferta ecuatoriana, que la completan los purés y pastas de frutas, madera y rosas.

Irán, gracias a su potencial en hidrocarburos, vende a Ecuador productos derivados de la refinación del petróleo tales como la parafina y la vaselina, conformando más del 70\% de la oferta iraní, complementada por 
productos lácteos y farmacéuticos. Existen asimismo identificados ciertos productos con potencial de intercambio: Productos primarios de la industria alimenticia en el caso de exportaciones ecuatorianas y bienes de capital y textiles por el lado de las importaciones (lacamara.org).

\section{Tabla 7.}

Principales productos de exportación e importación de Ecuador

\begin{tabular}{|c|c|c|}
\hline Exportaciones & \# & Importaciones \\
\hline \multirow{5}{*}{$\begin{array}{l}\text { Banano } \\
\text { Purés y pastas de frutas } \\
\text { Madera } \\
\text { Rosas } \\
\text { Potencial de Exportación }\end{array}$} & 1 & Parafina (Hidrocarburo) \\
\hline & 2 & Leche \\
\hline & 3 & Vaselina \\
\hline & 4 & Productos farmaceúticos \\
\hline & \# & Potencial de Importación \\
\hline \multirow{2}{*}{$\begin{array}{l}\text { Arroz } \\
\text { Aceite comestible }\end{array}$} & 1 & Maquinaria Agrícola \\
\hline & 2 & Textiles \\
\hline Maíz & 3 & Maquinaria de construcción \\
\hline Carne & 4 & Tecnología de plantas eléctricas \\
\hline
\end{tabular}

Fuente: (BCE \& Embajada de Irán en Ecuador)

\subsection{Principales productos exportados por Ecuador a Irán}

Entre las exportaciones que Ecuador envía hacia Irán, tenemos como producto estrella el banano $(96,76 \%)$ seguido por el café $(16,2 \%)$, el cacao $(1,3 \%)$ y frutos en general $(0,8 \%)$.

Tabla 8.

Principales productos exportados por Ecuador a Irán. Miles USD FOB

\begin{tabular}{clcc}
\hline Subpartidas & \multicolumn{1}{c}{ Descripción } & Año 2010 & Participación \\
\hline 0803.00 .12 .00 & Banano tipo (cavendish valery) & $8,049.07$ & $96.76 \%$ \\
\hline 2007.99 .92 .00 & Purés y pastas de frutas & 163.67 & $1.97 \%$ \\
\hline 4407.22 .00 .00 & Virola, imbuta y balsa & 85.15 & $1.03 \%$ \\
\hline 2008.99 .90 .00 & $\begin{array}{l}\text { Los demás frutas excepto } \\
\text { las mezclas }\end{array}$ & 18.40 & $0.23 \%$ \\
\hline 0603.11 .00 .00 & Rosas & 2.48 & $0.03 \%$ \\
\hline & \multicolumn{1}{r}{ Total general } & $\mathbf{8 , 3 1 8 . 7 6}$ & $\mathbf{1 0 0 . 0 0 \%}$ \\
\hline
\end{tabular}

Fuente: (Banco Central del Ecuador, 2010)

Elaboración: (PROECUADOR, Unidad de Inteligencia Comercial, 2010)

\subsection{Importaciones desde Irán a Ecuador}

Los productos que se importaron desde Irán en el 2010 son parafina, leche, vajillas, adoquines de granito, vaselina, cera de petróleo y micro cristalina en grupo.

\section{Tabla 9.}

Principales productos importados a Ecuador desde Irán: Miles de US FOB

\begin{tabular}{|c|c|c|c|}
\hline Subpartidas & Descripción & 2010 & Participación \\
\hline 2712.90 .30 .00 & $\begin{array}{l}\text { Parafina con un contenido de aco } \\
\text { igual a } 0,75 \% \text { en peso eite superior }\end{array}$ & 106.11 & $42.63 \%$ \\
\hline 1901.10 .10 .00 & Leche maternizada o humanizada & 53.15 & $21.35 \%$ \\
\hline 3921.10 .90 .00 & $\begin{array}{l}\text { Los demás vajillas y demás } \\
\text { artículos de uso doméstico y de } \\
\text { higiene. }\end{array}$ & 40.38 & $15.22 \%$ \\
\hline 6802.23 .00 .00 & Adoquines de granito & 29.26 & $11.76 \%$ \\
\hline 2712.10 .10 .00 & $\begin{array}{l}\text { Vaselina, parafina, cera de petróleo } \\
\text { micro cristalino en bruto }\end{array}$ & 18.81 & $7.56 \%$ \\
\hline 3002.90 .90 .00 & $\begin{array}{l}\text { Los demás antisueros (sueros con } \\
\text { anticuerpos) }\end{array}$ & 0.03 & $0.01 \%$ \\
\hline 4911.10 .00 .00 & $\begin{array}{l}\text { Impresos publicitarios, catálogos } \\
\text { comerciales y similares }\end{array}$ & 0.02 & $0.01 \%$ \\
\hline
\end{tabular}

Fuente: (Banco Central del Ecuador, 2010)

Elaboración: (PROECUADOR, Unidad de Inteligencia Comercial, 2010)

\subsection{Inversión extranjera directa de Irán en el Ecuador}

En el período 2000-2010 Irán registra poca inversión en el Ecuador. El valor contabilizado en el 2010 fue de 12.740 dólares. Una explicación a la reducida inversión es que no se ha concretado el financiamiento de ningún proyecto en el campo energético. Ecuador había mencionado que Irán pudiera financiar la construcción de la refinería del Pacífico, pero la iniciativa no se cristalizó. 
Tabla 10.

Inversión extranjera directa de Irán en el Ecuador años 2000 al 2010. Miles USD

\begin{tabular}{|c|c|c|c|c|c|c|c|c|c|c|c|c|}
\hline $\begin{array}{c}\text { Sectores } \\
\text { Inversiones }\end{array}$ & 2000 & 2001 & 2002 & 2003 & 2004 & 2005 & $2006^{*}$ & $2007 *$ & $2008 *$ & $2009^{*}$ & $2010^{*}$ & Total \\
\hline $\begin{array}{l}\text { Agricultura, silvicutura, } \\
\text { caza y pesca }\end{array}$ & - & - & - & - & - & - & - & - & - & - & - & 0,39 \\
\hline Comercio & - & - & 1,16 & - & - & - & - & - & 0,67 & 6,87 & - & 8,70 \\
\hline Industria manufacturera & - & - & - & - & - & - & - & - & - & - & 12,74 & 12,74 \\
\hline Total General & - & - & 1,16 & - & - & - & - & - & 0,67 & 6,87 & 12,74 & 21,83 \\
\hline
\end{tabular}

Fuente: (Banco Central del Ecuador, 2010)

Elaboración: (PROECUADOR, Unidad de Inteligencia Comercial, 2010)

\section{Conclusiones}

- Las relaciones comerciales entre Ecuador e Irán son prácticamente inexistentes. Esta afirmación se la puede comprobar al analizar los datos de la balanza comercial. En la balanza comercial no petrolera únicamente el año 2010 las exportaciones de Ecuador hacia Irán fueron algo significativas, alcanzando los 8'310.000 dólares. En los otros años (del 2008 al 2012) las exportaciones ecuatorianas son mínimas. En el año 2011 descienden a 1'186.000 y en el 2012 desaparecen totalmente. En cuanto a las importaciones que realiza nuestro país desde Irán, se debe indicar que en el período 20082012 -sumados todos los años- apenas llegan a 786.000 dólares.

- Analizando lo sucedido en la balanza comercial petrolera, la afirmación de la inexistencia de relaciones comerciales entre los dos países se reafirma. Siempre hablando del período 20082012 no existen exportaciones ecuatorianas hacia Irán, mientras que las importaciones son algo significativas solo en el año 2008 con 184'805.000 millones de dólares. Luego van descendiendo de año en año hasta desaparecer totalmente en el año 2012.

- Si se analiza desde el punto de vista de las inversiones los resultados son francamente decepcionantes. En el período 2000-2010 Irán invierte en el Ecuador la patética cifra de 21.830 dólares. Ecuador no invierte en Irán absolutamente nada.

- Refiriéndonos exclusivamente a Irán, se debe indicar que su mayor riqueza se encuentra más en sus reservas de gas que en las de petróleo. Efectivamente, según información proporcionada por Michael Klare, Irán posee un $15.5 \%$ de las reservas mundiales de gas, siendo las segundas reservas más grandes en el mundo, solo antecedidas por Rusia (país que ocupa el primer lugar). Debido a las reservas de hidrocarburos que posee Irán, a lo cual se suma su situación geográfica en el Mar Caspio y en el Golfo Pérsico, hacen de este país una de las regiones más explosivas, desde el punto de vista de la geopolítica, del planeta.

- A manera de conclusión general se debe indicar que Ecuador a futuro no podrá profundizar sus relaciones comerciales con Irán en vista de lo compleja que es la geopolítica iraní, a lo cual se suma la lejanía entre los dos países, lo cual encarece en extremo cualquier intercambio comercial. La intención de establecer relaciones comerciales entre los dos países más bien obedece a una estrategia de imagen por parte del gobierno ecuatoriano. 


\section{Referencias bibliográficas}

- AXWORTHY, M. (2010). Irán: Una historia desde Zoroastro hasta hoy. Ed. Turner Noema.

- Banco Central del Ecuador. (2010). BCE.

- BCE, \& Embajada de Irán en Ecuador. (s.f.). Principales productos de exportación e importación de Ecuador a Irán. Banco Central del Ecuador, Embajada de Irán en Ecuador.

- Central Bank of Iran. (2012). Banco Central de Irán, Exportaciones de crudo.

- CIA World Factbook. (2013).

- El Islam: Desde la caída de Constantinopla hasta nuestros días. (1975). España Editores.

- Enciclopedia Encarta. (2012). Mapa del Mar Caspio.

- Fondo Monetario Internacional (FMI). (2012). base de datos World Economic Outlook, Perspectiva de la economía mundial."

- http://es.wikipedia.org/wiki/Golfo_Persico. (s.f.). Obtenido de http://es.wikipedia.org/wiki/Golfo_Persico

- http://www.lacamara.org. (s.f.). Obtenido de http://www.lacamara.org/website/images/boletines/ informacion-comercial

- KLARE, M. (2008). Planeta Sediento, Recursos Menguantes: La nueva geopolítica de la energía. Editorial Tendencias.

- PROECUADOR. (2010). Unidad de Inteligencia Comercial.

- PROECUADOR. (2012). Ficha técnica Ecuador Iran.

- SERRATO, R. (2009). Desde el corazón de Irán: la esperanza oprimida. Erasmus Ediciones.

- SOOFI, A., \& GHAZINOORY, S. (2013). Science and Innovations in Iran: Development, Progress, and Challenges. Palgrave Macmillan. 